
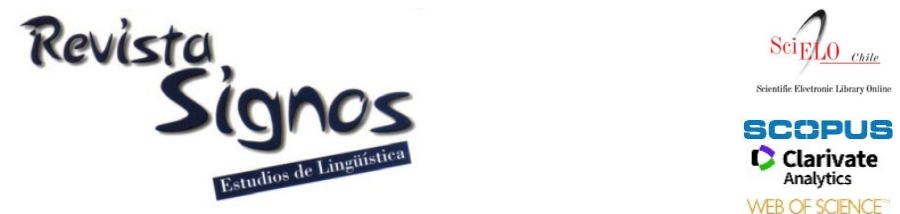

WEB OF SCIENCE

\title{
La formación de estudiantes universitarios para enseñar el español como segunda lengua a alumnos inmigrantes en España ${ }^{1}$
}

\section{The training of university students to teach Spanish as a second language to immigrant pupils in Spain}

\author{
Dimitrinka G. Níkleva \\ UNIVERSIDAD DE GRANADA \\ ESPAÑA \\ nikleva@ugr.es
}

\author{
Narciso M. Contreras-Izquierdo \\ UNIVERSIDAD DE JAÉN \\ ESPAÑA \\ ncontrer@ujaen.es
}

Recibido: 27-III-2017 / Aceptado: 22-VII-2019

DOI: $10.4067 /$ S0718-09342020000200496

\section{Resumen}

El tema de esta investigación es la formación y la competencia de los estudiantes universitarios (futuros docentes) para enseñar el español como segunda lengua (EL2) a alumnos inmigrantes en España. Los sujetos participantes en la investigación son 318 estudiantes universitarios, fundamentalmente del Grado de Educación Primaria y de Filología española, de distintas universidades: Granada, Almería, Jaén, Complutense de Madrid y Nebrija. El instrumento de investigación es un cuestionario compuesto por 58 ítems en el que se combinan preguntas cerradas y abiertas, diseñadas para los objetivos del estudio, o sea, para conocer la opinión y la valoración de la formación específica recibida, así como para medir y evaluar su capacidad para enseñar el español a alumnos inmigrantes. En el análisis de datos se aplican técnicas de análisis descriptivo de frecuencia y análisis inferencial para establecer la relación de dependencia significativa entre variables. Los resultados confirman el desajuste existente entre la formación de los futuros docentes y la realidad laboral, en la que se van a enfrentar a las dificultades y diferencias específicas en la atención a alumnado extranjero. Se trata de una laguna que urge subsanar, porque la competencia en español como lengua vehicular mejorará el rendimiento académico de los alumnos inmigrantes y, además, la competencia comunicativa en español es la vía más eficaz para la integración social y cultural de este colectivo. Se puede afirmar que no existen estudios previos que midan la capacidad real de los estudiantes universitarios para enseñar el español como segunda lengua. En este sentido, creemos que el presente estudio es pionero, además de relevante.

Palabras Clave: Formación universitaria, estudiantes universitarios, formación del profesorado de español como L2, español para inmigrantes, análisis estadístico cuantitativo con SPSS. 


\begin{abstract}
The subject of this study is the training and competence of university students (future teachers) to teach Spanish as a second language (EL2) to immigrant pupils in Spain. The study's participating subjects are 318 university students, mostly studying undergraduate degrees in Primary Education or Spanish Language \& Literature, at the universities of Granada, Almería, Jaén, Complutense of Madrid, and Nebrija. The research instrument employed is a questionnaire comprising 58 items, which combines closed and open questions designed for the purposes of this study, that is, to assess the specific training received for teaching Spanish to immigrant students. Data analysis is performed applying descriptive analysis of frequency and inferential analysis techniques in order to establish the significant relationship of dependence between variables. The results confirm the existing imbalance between the training of future teachers and the reality of work in which they will face specific difficulties and differences when teaching foreign students. This is a lack that must be corrected urgently, because competence in Spanish as a vehicular language improves the academic performance of immigrant students. Furthermore, communicative competence in Spanish is the most effective route to immigrants' social and cultural integration. There are no previous studies that measure the actual capability of university students to teach Spanish as a second language. In this regard, we believe that this study is pioneering as well as relevant.
\end{abstract}

Key Words: University training, university students, teacher training of Spanish as a second language, Spanish for immigrants, data analysis with the SPSS 21.

\title{
INTRODUCCIÓN
}

La población extranjera en España ha aumentado considerablemente en las últimas décadas, un hecho bien conocido, que ha originado múltiples medidas de atención y de resolución en todos los ámbitos: educativo, social, legislativo, sanitario, etc ${ }^{2}$. Entre todos se pretende la integración más rápida y eficaz de los inmigrantes y no cabe duda alguna de que la vía más rápida de integración social y cultural para los inmigrantes será la adquisición de una buena competencia comunicativa en español. Por tanto, es de suma importancia preparar a los profesores para que puedan desempeñar con éxito esta labor ${ }^{3}$. Sin embargo, nos atrevemos a afirmar que el sistema educativo sigue padeciendo un vacío en la formación de los futuros profesores de español y nos referimos a la formación específica necesaria para enseñar el español como segunda lengua a alumnos inmigrantes. Es cierto que se han diseñado varias medidas para afrontar la actual realidad multicultural y plurilingüe con la creación de aulas de apoyo lingüístico para los inmigrantes escolarizados, pero los planes de estudio de los futuros docentes que tendrán que enseñar el español como segunda lengua y como lengua vehicular a los alumnos inmigrantes no han sido adaptados a esta actualidad.

En las titulaciones universitarias de los futuros docentes de español (Grado en Educación Primaria y Grado en Filología española) no hay asignaturas específicas que 
les proporcionen la formación necesaria para adaptarse a esta situación, a pesar de que el ámbito formativo de la enseñanza y aprendizaje de lenguas está presente en los libros blancos que respaldan dichas titulaciones. En el libro blanco base de la titulación de Filología española (ANECA, 2005), la referencia al español para extranjeros e inmigrantes incluso aparece recogida expresamente en la definición de los objetivos:

"El ámbito profesional preferente es el de la docencia, tanto como primera lengua, como -más recientemente- lengua para extranjeros, atendiendo a la realidad de las nuevas poblaciones de inmigrantes, y de demanda en otros países" (ANECA, 2005: 433).

Por tanto, y a nuestro entender, en dichas titulaciones deberían realizarse modificaciones curriculares para incluir de forma efectiva contenidos formativos específicos en el ámbito de la enseñanza y aprendizaje del español como segunda lengua y como lengua vehicular a los alumnos inmigrantes. En el caso que nos ocupa, el profesor necesita, además de una formación general en lingüística aplicada y didáctica de lenguas, una específica sobre enseñanza a inmigrantes.

Por todo ello, hemos diseñado este estudio para conocer la opinión y la valoración de estudiantes universitarios sobre su formación, evaluar y medir su capacidad y concienciar sobre la imperante necesidad de incluir estos contenidos específicos en las titulaciones universitarias y no dejarlos solo para los másteres especializados.

\section{Estudios precedentes}

Después de haber realizado una amplia búsqueda bibliográfica, creemos que podemos afirmar que no existen estudios previos que midan la capacidad real de los estudiantes universitarios para enseñar el español a alumnos no nativos. En este sentido, nuestro estudio es pionero, y por tanto, consideramos que relevante. Sí existen otros estudios sobre la presencia o la ausencia de esta formación en los planes de estudio como el de Cruz Piñol (2004) y el de Pastor Cesteros (2007), que abarca tanto los estudios de grado como de posgrado. Estos dos estudios señalan que desde principios de los años noventa aumentan las facultades que ofrecen en sus planes de estudio alguna asignatura (normalmente optativa) relacionada con el español como lengua extranjera. Se trata de las siguientes universidades: de Barcelona, Oviedo, Autónoma de Madrid, Zaragoza, Salamanca, UNED, Valencia, Pablo de Olavide y Málaga (Cruz Piñol, 2004). A ellas, Pastor Cesteros (2007) añade otras universidades como la Universidad de Santiago de Compostela, la Universidad de Vigo, la Universidad de La Coruña, la Universidad de León, la Universidad Autónoma de Barcelona y la Universidad de Alicante (que dispone de la 'Mención de Español como Lengua Extranjera', dentro de la titulación de Filología Hispánica, con al menos seis asignaturas sobre la enseñanza del español para extranjeros). 
El estudio más actual sobre este tema en los planes de estudio es el de Rico-Martín y Níkleva (2017). Se centra en la Comunidad Autónoma de Andalucía y abarca ocho universidades andaluzas (las de Almería, Cádiz, Córdoba, Granada, Huelva, Jaén, Málaga y Sevilla). En todos estos centros universitarios se imparte alguna materia relacionada de alguna forma con la atención a la diversidad, pero son escasos los contenidos dedicados a la enseñanza del EL2 para inmigrantes. Se puede suponer por la vinculación de estas materias con los distintos departamentos. En Granada, los dos Departamentos encargados de impartir las asignaturas básicas señaladas son Didáctica y Organización Escolar junto con Psicología Evolutiva y de la Educación, mientras que en Sevilla se adscriben a los Departamentos de Didáctica y Organización Educativa, Métodos de Investigación y Diagnóstico en Educación.

Las asignaturas de carácter didáctico-disciplinar son responsabilidad de los Departamentos de Didáctica de la Lengua y la Literatura en Cádiz y de Filología Española en Jaén.

Respecto a las asignaturas optativas, en Cádiz se adscriben al Departamento de Didáctica de la Lengua y la Literatura, igual que en la Facultad de Granada, pero la optativa de la Facultad de Melilla pertenece al Departamento de Psicología Evolutiva y de la Educación. En Córdoba las asignaturas están adscritas a tres Departamentos (Matemáticas, Filología Inglesa y Alemana, y Psicología Evolutiva y de la Educación). Las optativas de la Facultad de Huelva, así como todas las de Málaga y Sevilla, pertenecen a Didáctica y Organización Escolar/Educativa. Por último, en Jaén pertenecen a los Departamentos de Filología española, Filología Inglesa y Lenguas y culturas mediterráneas.

Este estudio confirma la carencia de formación específica para enseñar la lengua y la cultura española a alumnos inmigrantes. La formación recibida, insuficiente y relacionada con la diversidad cultural, es fundamentalmente de tipo optativo, lo que no garantiza que todos los estudiantes cursen estas asignaturas y tengan estos conocimientos. Además, se trabaja la diversidad junto a otras necesidades educativas especiales en el ámbito de la denominada educación inclusiva, pero sin contenidos lingüísticos específicos.

A pesar de que Pastor Cesteros (2007) se planteaba la duda de si se podía pedir a un grado universitario una especialización que no tenía la obligación de ofrecer por corresponder más bien a los estudios de posgrado, nosotros tendemos a considerar que sí es necesario porque cuando nuestros estudiantes universitarios empiecen a trabajar como profesores comprobarán que la presencia de alumnado extranjero en las aulas de Educación Primaria y Secundaria no será optativa (como las asignaturas), sino sencillamente real. Por tanto, resulta absolutamente necesario, si no obligatorio, disponer de la formación específica para poder enseñar el español a alumnos no nativos. 
En la titulación del Grado de Educación Primaria los alumnos tienen la asignatura de prácticas en los colegios y allí tienen su primer contacto con esta realidad y con las dificultades específicas que les plantea. Los de Filología española no tienen prácticas profesionales durante la carrera, sino posteriormente, en el caso de realizar algún máster, normalmente el que les capacita para ser profesores en Educación Secundaria. Mencionamos este hecho para tenerlo en cuenta a la hora de valorar los datos del cuestionario.

Hay otros tipos de estudios relacionados con la legislación (Grañeras, Vázquez, Parra, Rodríguez, Madrigal \& Vale, 2007), las aulas de apoyo lingüístico para inmigrantes (García, Sánchez, Moreno \& Goenechea, 2010; Níkleva \& Ortega, 2015, entre otros), las necesidades de los docentes (Moreno García, 2007), numerosas investigaciones sobre interculturalidad, la inmensa investigación de Villalba Martínez y Hernández García (2008), etc. Sin embargo, todos los estudios que hemos mencionado son muy distintos del nuestro temáticamente: analizan solo los planes de estudio, las medidas de atención y las aulas de apoyo lingüístico, la interculturalidad, etc. pero no cuentan con los estudiantes como participantes y con sus conocimientos reales sobre el español para inmigrantes. Nuestro estudio es el primero que pretende valorar los conocimientos adquiridos mediante la formación universitaria recibida para así poder formular conclusiones fundamentadas sobre la necesidad de mejorar la formación específica en las titulaciones universitarias.

\section{Objetivos de la investigación}

Esta investigación se enmarca en un proyecto $\mathrm{I}+\mathrm{D}$ que parte de la necesidad de ajustar la formación académica de los docentes a la demanda laboral y a las características de la actual sociedad española, entre las que destaca el número elevado de inmigrantes, por lo que dicho proyecto se centra en la formación de los docentes para enseñar el español como segunda lengua a dicho colectivo.

De este modo, de acuerdo con lo expuesto anteriormente, y en el marco del proyecto $\mathrm{I}+\mathrm{D}$, para esta investigación nos hemos planteado los siguientes objetivos.

\subsection{Objetivos generales}

- Conocer la opinión y la valoración que un grupo de estudiantes realiza respecto de su formación recibida en la universidad para atender a inmigrantes.

- Evaluar la capacidad real de los estudiantes universitarios para enseñar el español como segunda lengua (EL2).

- Medir su competencia sobre conceptos y contenidos concretos del ámbito del EL2. 


\subsection{Objetivos específicos}

- Averiguar si los estudiantes universitarios se consideran preparados para enseñar español a alumnos inmigrantes como futuros docentes, concretamente en las áreas de formación lingüística, dominio de una lengua extranjera y dominio de la lengua objeto de estudio de los inmigrantes, y si necesitan y reclaman más formación específica.

- Analizar la posible experiencia docente en EL2, y más específicamente con inmigrantes, así como las posibles dificultades que han encontrado en dicha labor.

- Medir sus conocimientos en ámbitos como documentos curriculares (MCER y PCIC), adaptación curricular, aulas ATAL, niveles de aprendizaje, factores que dificultan la enseñanza del EL2, funciones comunicativas, discurso del profesor, contenidos en la enseñanza del EL2, así como su dominio de la categoría gramatical, especialmente del verbo (modos indicativo, subjuntivo e imperativo; pretérito indefinido e imperfecto; estructura regular e irregular; facilidad y complejidad en su enseñanza).

- Analizar la posible relación significativa entre las variables presentes en el estudio (titulación, conocimientos y conceptos fundamentales en EL2).

\section{Metodología de la investigación}

A continuación presentamos el diseño de la investigación: método y técnicas de análisis, estudio piloto, participantes, instrumento y variables de investigación, análisis de datos y discusión de los resultados.

\subsection{Método de investigación}

El método de esta investigación es cuantitativo (descriptivo e inferencial). Las variables son nominales (cualitativas) predominantemente, además de ordinales (escala Likert). Una vez establecidos los objetivos del estudio, el proceso se dividió en las siguientes etapas: 1) preparación del instrumento de investigación; 2) un estudio piloto para comprobar la validez del diseño metodológico; 3) codificación y procesamiento de datos; 4) análisis estadístico de los datos mediante diferentes técnicas; 5) análisis de los datos y discusión de los resultados y 6) obtención de conclusiones.

El análisis de datos se realizó con el programa estadístico SPSS 21 (Statistical Product and Service Solutions). Se realizó el análisis descriptivo de las variables individuales mediante tablas de frecuencia. Las preguntas de escala Likert fueron evaluadas según los estadísticos de centralidad (moda, mediana y media), mientras que para las preguntas categóricas se realizaron tablas de frecuencia y gráficos de sectores. Para el estudio de la relación entre variables cualitativas se han empleado tablas de contingencia con la prueba de independencia Chi-cuadrado y la prueba exacta de 
Fisher para analizar si existe o no relación entre las variables. En los casos donde los contrastes fueron significativos se midió la fuerza de asociación mediante el coeficiente $\mathrm{V}$ de Cramer. Se tomó un nivel de significación del 5\% para todas las pruebas.

Tal como hemos señalado anteriormente se llevó a cabo un estudio piloto para probar la pertinencia de la metodología utilizada, sobre todo, para probar el cuestionario, su comprensión, extensión, las variables y su codificación mediante etiquetas en SPSS. El estudio piloto se realizó con 68 alumnos. De acuerdo con los resultados obtenidos se hicieron las siguientes modificaciones y reflexiones:

- Las respuestas a las preguntas abiertas se codificaron como 'bien', 'regular' y 'mal' o como 'bien' y 'mal' para reducir el número de categorías para el análisis. Las respuestas libres fueron muy variadas, pero se tomó la decisión de valorarlas en función de la corrección de la respuesta.

- Había dos preguntas sobre tiempos verbales: ¿cuál es el más difícil y el más fácil morfológicamente para un extranjero? En el estudio piloto codificamos las respuestas como 'bien' y 'mal' o 'verdadero' y 'falso', pero decidimos para el estudio posterior asignar 18 etiquetas para todos los tiempos verbales para tener más información respecto a la opinión de los alumnos. En estas dos preguntas se confirmó nuestra hipótesis de que los estudiantes no entienden bien la pregunta, pero decidimos no modificarla para el estudio posterior, puesto que no era un fallo en la formulación de la pregunta, sino que se trataba de desconocimiento por parte de los sujetos. La no comprensión de la pregunta, que por sí misma revela escasa preparación, provocó muchas respuestas equivocadas en el estudio piloto (78,3\% sobre el tiempo más difícil y 100\% sobre el tiempo más fácil), pero decidimos dejar la pregunta con la misma formulación, precisamente porque en nuestra opinión mide de una manera muy acertada la conciencia metalingüística y la preparación para enseñar el español a inmigrantes.

- El cuestionario es más largo de lo recomendable, pero aun así decidimos dejar el mismo número de preguntas para poder recabar toda la información que necesitábamos. El requisito estadístico, según el cual por cada ítem del cuestionario debe haber tres sujetos como mínimo, está cumplido, puesto que disponemos de 318 sujetos para 58 ítems.

\subsection{Participantes}

Los sujetos participantes en la investigación son 318 estudiantes universitarios de distintas universidades: Granada, Almería, Jaén, la Complutense de Madrid y Nebrija.

Solo un 4,8\% de los sujetos ya posee un título universitario. La mayoría son estudiantes en el Grado de Educación Primaria (70,8\%), un 28,9\% son de Filología española y un $0,3 \%$ estudian en otras titulaciones. Están representados todos los 
cursos de cada titulación, pero predominan los de $3^{\circ}$ y $4^{\circ}$, ambos representados con un $39 \%$ cada uno.

La edad de los participantes se ha distribuido por grupos y la moda es del grupo de 17-25 años formado por un 88,7\%. En cuanto al sexo, predominan las mujeres con un $71,1 \%$ frente a un $28,9 \%$ para los hombres. El 94\% son de nacionalidad española frente a un $6 \%$ de extranjeros. El 79,6\% de los estudiantes ha vivido solo en España.

\subsection{Instrumento y variables}

El instrumento de investigación es un cuestionario compuesto por 58 ítems en el que se combinan preguntas cerradas y abiertas ${ }^{4}$. El cuestionario se contestó de manera presencial $(91,2 \%)$ y online $(8,8 \%)$. La recogida de datos garantizó el anonimato de las personas que lo han respondido, lo que aseguró la confidencialidad de la información recabada. El primer bloque de preguntas estuvo dedicado a datos de identificación, donde se pidió a los alumnos que indicasen su edad, sexo, Universidad, curso en el que están matriculados y titulación que cursaban, su nacionalidad y la de los padres.

Una de las preguntas se refiere a si han vivido fuera de España, puesto que consideramos que este dato puede influir en algunas de las respuestas. Hay un bloque de ocho preguntas de tipo escala Likert para medir el grado de acuerdo o desacuerdo con una serie de afirmaciones. El siguiente bloque de preguntas cerradas y abiertas se diseñó para seguir respondiendo a los objetivos del estudio y medir la formación recibida para impartir el español a alumnos inmigrantes.

En cuanto a la fiabilidad y la validez del cuestionario, hemos obtenido los siguientes valores. La fiabilidad se confirma con el alfa de Cronbach de 0,73 lo que indica consistencia interna entre los datos, mientras que la validez del instrumento se comprueba con los valores de KMO (Kaiser-Meyer-Olkin) de 0,71 y el test de Bartlett con el valor de 0,03 .

\section{Análisis de datos y discusión de resultados}

De acuerdo con el tipo de variables y grupos de sujetos, se aplicaron dos técnicas de análisis cuantitativo: descriptivo e inferencial.

\subsection{Resultados del análisis descriptivo}

En el cuestionario hay dos preguntas sobre las siglas de las dos fuentes bibliográficas por excelencia que debería conocer cualquier profesor o investigador en el ámbito del español como lengua extranjera. Los resultados confirman que los estudiantes las conocen: el 91,8 conoce la sigla MCER (Marco común europeo de referencia para las lenguas) y el 95,9\% conoce la sigla PCIC (Plan curricular del Instituto Cervantes). 
A continuación (Tabla 1) presentamos los resultados de las preguntas tipo escala Likert, donde $1=$ muy en desacuerdo; 2 = algo en desacuerdo; $3=$ ni acuerdo ni desacuerdo (indiferente); $4=$ algo de acuerdo y $5=$ muy de acuerdo.

Tabla 1. Respuestas a las preguntas de escala Likert.

\begin{tabular}{|c|l|c|c|c|c|c|}
\hline $\mathbf{N .}^{\cdot}$ & \multicolumn{1}{|c|}{ PREGUNTAS } & $\mathbf{1}$ & $\mathbf{2}$ & $\mathbf{3}$ & $\mathbf{4}$ & $\mathbf{5}$ \\
\hline 12 & $\begin{array}{l}\text { Te consideras preparado para enseñar español a } \\
\text { alumnos inmigrantes como futuro docente. }\end{array}$ & $2,5 \%$ & $10,1 \%$ & $22,3 \%$ & $43,1 \%$ & $22 \%$ \\
\hline 13 & $\begin{array}{l}\text { La dificultad de enseñar español a alumnos } \\
\text { inmigrantes es mayor (en comparación con la } \\
\text { enseñanza del español a alumnos nativos) }\end{array}$ & $4,1 \%$ & $10,7 \%$ & $16,4 \%$ & $39,4 \%$ & $29,3 \%$ \\
\hline 14 & $\begin{array}{l}\text { La formación en enseñanza del español como } \\
\text { segunda lengua (L2) o para inmigrantes puede } \\
\text { resultar importante para tu futuro laboral. }\end{array}$ & $0,6 \%$ & $2,2 \%$ & $13,5 \%$ & $38,7 \%$ & $43,7 \%$ \\
\hline 15 & $\begin{array}{l}\text { Tienes suficiente formación específica lingǘrtica } \\
\text { para la enseñanza del español como lengua } \\
\text { extranjera y como segunda lengua. }\end{array}$ & $5,1 \%$ & $15,2 \%$ & $33,2 \%$ & $36,1 \%$ & $10,4 \%$ \\
\hline 16 & $\begin{array}{l}\text { Es necesario dominar la lengua nativa del alumno } \\
\text { inmigrante para que la clase sea efectiva. }\end{array}$ & $6,3 \%$ & $23,8 \%$ & $20,6 \%$ & $31,4 \%$ & $17,8 \%$ \\
\hline 17 & $\begin{array}{l}\text { Es útil el conocimiento de alguna lengua extranjera } \\
\text { para enseñar a alumnos inmigrantes. }\end{array}$ & $0,6 \%$ & $4,7 \%$ & $15,8 \%$ & $44,6 \%$ & $34,2 \%$ \\
\hline 18 & $\begin{array}{l}\text { Cuando seas docente, preferirás trabajar con los } \\
\text { alumnos inmigrantes fuera del aula ordinaria, en un } \\
\text { aula de apoyo lingüístico. }\end{array}$ & $10,8 \%$ & $16,8 \%$ & $38,6 \%$ & $25,3 \%$ & $8,5 \%$ \\
\hline 19 & $\begin{array}{l}\text { Cuando seas docente, preferirás trabajar con los } \\
\text { alumnos inmigrantes dentro del aula ordinaria } \\
\text { (junto con los alumnos nativos). }\end{array}$ & $6 \%$ & $13 \%$ & $32 \%$ & $32 \%$ & $17,1 \%$ \\
\hline
\end{tabular}

De los resultados anteriores hemos de destacar que a la pregunta 12 'Te consideras preparado para enseñar español a alumnos inmigrantes como futuro docente' los alumnos que contestan 'muy de acuerdo' y 'algo de acuerdo' suman un 65,1\% frente a un solo 2,5\% que responden 'muy en desacuerdo'. Esta autoevaluación alta que se atribuyen no se confirma en el resto de las respuestas que realmente miden su preparación.

A la pregunta 15 sobre la formación específica lingüística para la enseñanza del español como lengua extranjera y como segunda lengua, las respuestas 'algo de acuerdo' y 'muy de acuerdo' suman el 46,5\%.

De los resultados se observa que predomina la preferencia de trabajar con los alumnos inmigrantes dentro del aula ordinaria (junto con los alumnos nativos): un $49,1 \%$ frente a un 33,8 que preferirían hacerlo fuera aula ordinaria, en un aula de apoyo lingüístico.

El 73,2\% considera que durante la carrera universitaria sí ha recibido formación general para atender a alumnos inmigrantes y el 79\% que ha recibido formación específica lingüística para enseñar español a inmigrantes. El 51,8\% afirma haber recibido esta formación en una asignatura específica, pero el 73,35\% la considera 
insuficiente. De los que no han recibido esta formación, un porcentaje muy alto $(92,1 \%)$ considera que le hace falta.

Para la mejor interpretación de los resultados hemos querido comprobar si los estudiantes tienen experiencia docente. El 18,3\% no tiene ninguna experiencia docente. El resto se distribuye de la siguiente manera: prácticas en colegios en la titulación de Magisterio (26,9\%), clases particulares (21,2\%), academias $(2,2 \%)$ y varias de las anteriores $(31,4 \%)$. De todos ellos, el 53,7\% ha tenido experiencia con alumnos inmigrantes y el 30\% reconoce haber tenido dificultades para atenderlos. Enumeran las dificultades encontradas de la siguiente manera (Gráfico 1):

28. En caso afirmativo, ¿de qué tipo han sido las dificultades?

$\begin{array}{ll}\square \text { Comprensión y expresión oral } & \square \text { Otro } \\ \square \text { Comprensión y expresión escrita } & \square \text { Diferencias culturales } \\ \square \text { Lectoescritura } & \square \text { Disciplina }\end{array}$

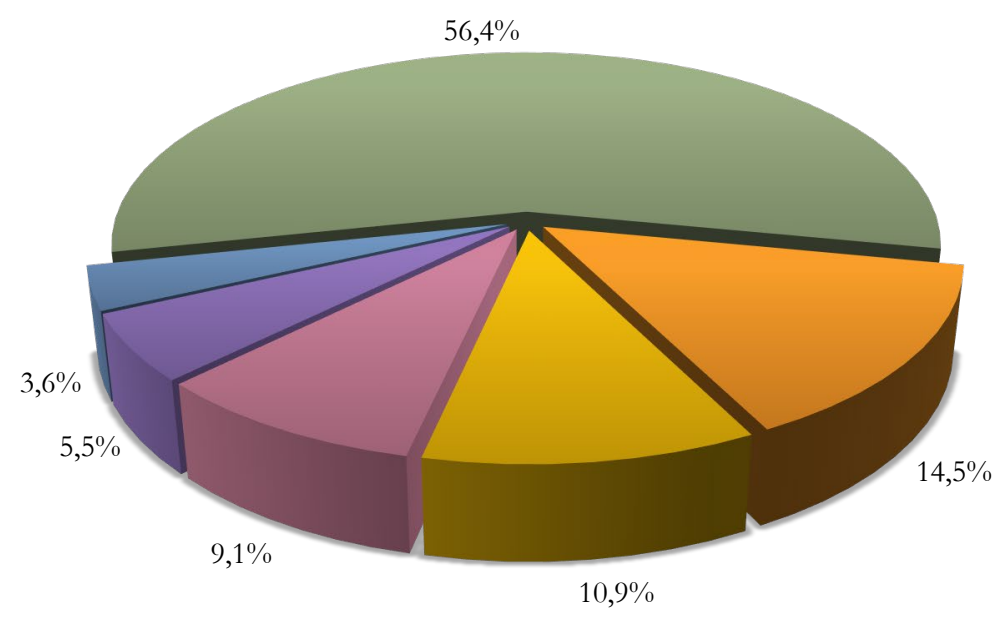

Gráfico 1. Principal dificultad de los estudiantes universitarios en la atención a alumnos inmigrantes.

En el Gráfico 1 se observa que la principal dificultad según los sujetos encuestados es la comprensión y expresión oral $(56,4 \%)$.

El 81,5\% afirma que necesitaba algún tipo de formación que no había recibido todavía en su carrera para sentirse más seguro y competente a la hora de impartir clases.

Para nuestra investigación era importante comprobar si los estudiantes conocen las aulas ATAL (Aulas Temporales de Adaptación Lingüística) que son las aulas de apoyo lingüístico para alumnos inmigrantes con nivel inferior a B1 en Andalucía. En otra pregunta se incluyeron las aulas de enlace (en Madrid) por los sujetos que no estudian 
en Andalucía. El 80,3\% de los sujetos encuestados reconoce no conocer estas aulas. Aun así, en una pregunta posterior se les pregunta qué es el aula ATAL y las respuestas se distribuyen de la siguiente manera: solo el 28,9\% contesta correctamente; el resto considera que son aulas temporales de asesoramiento legal para alumnos inmigrantes $(35,2 \%)$ o aulas de apoyo e-learning para alumnos inmigrantes (26,7\%). De la misma manera, el 97,2\% afirma conocer las aulas de enlace, pero se confirma que es así solo para el 47,6\%.

El 83,8\% sabe en qué consiste una adaptación curricular. Saben también quién la elabora $(91,4 \%)$ y para quién se hace $(83,2 \%)$.

Nos interesaba también saber si los estudiantes participantes en el estudio tienen un nivel B1 en una lengua extranjera. Los resultados confirman que el 58,4\% sí tienen el B1.

Tal como hemos explicado anteriormente, en nuestra investigación hemos querido comprobar si los estudiantes tienen conocimientos sobre los conceptos fundamentales en la enseñanza del español como segunda lengua. Sorprendentemente, a una pregunta tan elemental como cuáles son los niveles de referencia de una lengua extranjera, cuya respuesta sería de A1 a C2, solo el 80,9\% contesta bien (las respuestas han sido categorizadas como 'bien', 'mal' y 'regular').

A la pregunta de cuál es el primer modo verbal que enseñarían a los alumnos inmigrantes contestan 'bien' el 86,1\% de los estudiantes. Aunque el porcentaje es alto, consideramos que si los alumnos tuvieran conocimientos muy básicos de gramática y de la especificidad del español como lengua extranjera, contestarían correctamente en su totalidad: empezar por el modo indicativo y no por el imperativo o el subjuntivo. Además, el 17,8\% de los estudiantes demuestran no distinguir entre verbos regulares e irregulares y no acertar en su decisión de cuál es el primer verbo regular o irregular que enseñarían en el nivel A1: cuando se les pregunta el ejemplo del verbo regular contestan con alguno irregular y viceversa.

Teníamos especial interés en dos preguntas, cuyas respuestas revelan la experiencia y la conciencia sobre las dificultades para un extranjero: cuál es el tiempo verbal más fácil y el más difícil morfológicamente. En primer lugar, tal como confirmó el estudio piloto los estudiantes universitarios ni siquiera entienden bien la pregunta: 'morfológicamente' se refiere a la formación/la conjugación del verbo en un tiempo y las irregularidades que presenta. Si hubiéramos planteado la pregunta sobre la dificultad funcional, o sea de uso, las respuestas deberían indicar cualquier tiempo del modo subjuntivo. En nuestra opinión, el tiempo más fácil morfológicamente es el imperfecto de indicativo, porque tiene solo tres verbos irregulares: 'ser', 'ir' y 'ver'. Todos los demás se comportan como regulares y, además, la segunda y la tercera conjugación coinciden y esto representa otra facilidad añadida. En cuanto al tiempo más difícil morfológicamente, nosotros daríamos dos respuestas: el pretérito perfecto 
simple (indefinido) de indicativo o el presente de indicativo. La percepción de los extranjeros señala el pretérito perfecto simple (indefinido) de indicativo. Quizás influya también el hecho de que el presente de indicativo es el primero que aprenden y la mayor frecuencia de su uso, por lo que lo perciben como más fácil. Hemos considerado como respuesta correcta los dos tiempos, aunque nosotros personalmente nos inclinamos por el presente de indicativo.

A continuación recordamos, para ilustrar nuestra opinión, las irregularidades en Presente de Indicativo y en Pretérito perfecto simple de Indicativo con algunos ejemplos (Tabla 2 y Tabla 3).

Tabla 2. Verbos de conjugación irregular en Presente de Indicativo.

\begin{tabular}{|c|c|c|}
\hline \multicolumn{3}{|c|}{ Verbos con irregularidad propia } \\
\hline \multirow{2}{*}{\multicolumn{3}{|c|}{$\begin{array}{l}\text { Ejemplos: 'ser', 'estar', 'decir', 'hacer', 'tener', 'ir', 'poner', 'salir', 'caber', 'traer', 'saber', 'oír', etc. } \\
\text { Verbos con irregularidad común }\end{array}$}} \\
\hline & & \\
\hline Grupo & Cambio & Ejemplos \\
\hline \multirow[t]{2}{*}{$\begin{array}{l}\text { Verbos en -ar con diptongación } \\
\left(1 .^{\mathrm{a}}, 2 .^{\mathrm{a}}, 3 .^{\mathrm{a}} \text { pers. sing. } 3 \text { 3. }^{\mathrm{a}} \text { pers. plural }\right)\end{array}$} & e $>$ ie & $\begin{array}{l}\text { 'pensar' > 'pienso' } \\
\text { 'comenzar' > 'comienzo' } \\
\text { 'empezar' > 'empiezo' }\end{array}$ \\
\hline & o> ue & 'jugar' > 'juego' \\
\hline \multirow[t]{2}{*}{$\begin{array}{l}\text { Verbos en -er con diptongación } \\
\left(1 .^{a}, 2 .^{a}, 3 .^{a} \text { pers. sing.; } 3 .^{a} \text { pers. plural }\right)\end{array}$} & $\mathrm{e}>\mathrm{ie}$ & $\begin{array}{l}\text { 'querer' > 'quiero' } \\
\text { 'entender' > 'entiendo' } \\
\text { 'defender' > 'defiendo' } \\
\text { 'perder' > 'pierdo' }\end{array}$ \\
\hline & o $>$ ue & $\begin{array}{l}\text { 'poder' > 'puedo' } \\
\text { 'volver' > 'vuelvo' }\end{array}$ \\
\hline \multirow{3}{*}{$\begin{array}{l}\text { Verbos en }- \text { ir con cambio vocálico } \\
\left(1 .^{a}, 2 .^{a}, 3 .^{a} \text { pers. sing.; } 3 .^{a} \text { pers. plural }\right)\end{array}$} & $e>i$ & 'pedir' > 'pido' \\
\hline & e $>$ ie & 'preferir' > 'prefiero' \\
\hline & o> ue & 'dormir' > 'duermo' \\
\hline $\begin{array}{l}\text { Verbos con cambio consonántico } \\
\text { (1. }{ }^{\text {a }} \text { persona singular) }\end{array}$ & $\mathrm{c}>\mathrm{zc}$ & $\begin{array}{l}\text { 'conocer' > 'conozco' } \\
\text { 'traducir' > 'traduzco' }\end{array}$ \\
\hline
\end{tabular}

Como se observa en la tabla, el hecho de que el cambio se produce solo en algunas personas gramaticales y no en todas supone una dificultad más para los estudiantes extranjeros.

Recordemos también las irregularidades en Pretérito perfecto simple de Indicativo (conocido también como Indefinido) (Tabla 3). 
Tabla 3. Verbos de conjugación irregular en Pretérito Perfecto Simple de Indicativo.

\begin{tabular}{|c|c|}
\hline Grupo de irregularidad & Ejemplos \\
\hline De irregularidad propia & 'ser', 'estar', 'hacer', 'ir', 'dar', 'poner', 'poder', 'tener', 'venir' \\
\hline Pretéritos con 'u' & $\begin{array}{l}\text { 'estar' > 'estuve', 'poder' > 'pude', 'poner' > 'puse', 'tener' > 'tuve', } \\
\text { 'saber' > 'supe', 'caber' > 'cupe', 'andar' > 'anduve', 'haber' > } \\
\text { 'hube' }\end{array}$ \\
\hline Pretéritos con 'j' & $\begin{array}{l}\text { 'decir' > 'dije', 'traer' > 'traje', 'conducir' > 'conduje', 'traducir' > } \\
\text { 'traduje', 'producir' > 'produje', 'reducir' > 'reduje', 'deducir' > } \\
\text { 'deduje' }\end{array}$ \\
\hline Pretéritos con ' $\mathrm{i}$ ' & $\begin{array}{l}\text { 'hacer' > 'hice', 'querer' > 'quise', 'venir' > 'vine', 'convenir' > } \\
\text { 'convine' }\end{array}$ \\
\hline $\begin{array}{l}\text { Con cambio vocálico e }>\mathrm{i} \\
\text { (excepto delante de -i- tónica) }\end{array}$ & $\begin{array}{l}\text { 'pedir' > 'pidió', 'vestir' > 'vistió', 'elegir' > 'eligió', 'corregir' > } \\
\text { 'corrigió, 'servir' > 'sirvió', 'seguir' > 'siguió', 'impedir' > 'impidió', } \\
\text { 'medir' > 'midió' }\end{array}$ \\
\hline $\begin{array}{l}\text { Con cambio vocálico o>u } \\
\text { (excepto delante de -i- tónica) }\end{array}$ & 'dormir' > 'durmió', 'morir' > 'murió' \\
\hline con vocal al final del radical & 'leer' > 'leyó', 'leyeron' \\
\hline
\end{tabular}

Siguiendo con el cuestionario y el análisis de datos, hemos de aclarar que a los estudiantes participantes en el estudio no se les proporcionaron los nombres de los tiempos verbales para que eligieran entre ellos con el objetivo de no ayudar a los estudiantes en este aspecto y para que tuvieran que decir el nombre de un tiempo ellos mismos, explicar las razones por las que lo consideran el más fácil o el más difícil morfológicamente y poner un ejemplo de verbo conjugado en este tiempo.

Hemos de aclarar que muchos de los estudiantes no contestan con el nombre de un tiempo verbal (que es lo que se pide en la pregunta), sino ponen un ejemplo: 'hablo', 'hube hablado', etc. Nosotros no hemos descartado estas respuestas (a pesar de plantearnos esta opción), sino que hemos completado el nombre del tiempo. Otros dicen nombres inexistentes como Presente simple o Pasado simple (estas respuestas han sido categorizadas como 'otro').

A continuación presentamos la forma de codificar las respuestas que se distribuyen de la siguiente manera (Tabla 4). 
Tabla 4. Respuestas a la pregunta sobre el tiempo verbal más difícil morfológicamente.

\begin{tabular}{|l|c|}
\hline \multicolumn{1}{|c|}{ Tiempo verbal } & Porcentaje \% \\
\hline Formas simples de Indicativo & 27 \\
\hline 1 = Presente de Indicativo ('hablo') & 1,6 \\
\hline 2 = Futuro simple de Indicativo ('hablaré') & 8,2 \\
\hline 3 = Pretérito perfecto simple (Indefinido) de Indicativo ('hablé') & 1,6 \\
\hline 4 = Pretérito Imperfecto de Indicativo ('hablaba') & 9 \\
\hline $5=$ Condicional simple de Indicativo ('hablaría') & 1,3 \\
\hline Formas compuestas de Indicativo & 4,1 \\
\hline 6 = Pretérito Perfecto Compuesto de Indicativo ('he hablado') & 5 \\
\hline $7=$ Pretérito Pluscuamperfecto de Indicativo ('había hablado') & 0,3 \\
\hline $8=$ Pretérito anterior de Indicativo ('hube hablado') & 0,3 \\
\hline $9=$ Futuro perfecto o compuesto de Indicativo ('habré hablado') & 10,7 \\
\hline $10=$ Condicional compuesto o perfecto Indicativo ('habría hablado') & 9,1 \\
\hline Formas simples de Subjuntivo & 2,5 \\
\hline $11=$ Presente de Subjuntivo ('hable') & 1,3 \\
\hline $12=$ Pretérito imperfecto de Subjuntivo ('hablara' o 'hablase') & 13,2 \\
\hline $13=$ Futuro de Subjuntivo ('hablare') & 1,3 \\
\hline Formas compuestas de Subjuntivo & 0,6 \\
\hline $14=$ Pretérito perfecto compuesto de Subjuntivo ('haya hablado') & \\
\hline $15=$ Pretérito pluscuamperfecto de Subjuntivo ('hubiera/se hablado') & 10,4 \\
\hline $16=$ Futuro Perfecto de Subjuntivo ('hubiere hablado') & \\
\hline $\begin{array}{l}17=\text { Modo Imperativo } \\
\text { 'habla', 'hablad' }\end{array}$ & \\
\hline $18=$ otro (mal; nombre inexistente de tiempo verbal) & \\
\hline
\end{tabular}

En la Tabla 4 hemos etiquetado con el número 18 las respuestas incorrectas sobre un nombre inexistente de tiempo verbal en español. En cuanto al resto de respuestas, el porcentaje más alto $(27 \%)$ señala el Presente de Indicativo como el tiempo más difícil morfológicamente y en este sentido coincide con nuestra opinión. Al Pretérito perfecto simple le corresponde un 8,2\%. Muchos estudiantes señalan el Presente de Subjuntivo $(10,7 \%)$ por la dificultad de usarlo, a pesar de que esto no representa una dificultad morfológica, sino funcional. Nos parece preocupante que un 10,4\% de los estudiantes universitarios señalan un tiempo inexistente en español.

Un 38,7\% han contestado 'bien' la pregunta sobre las razones para considerarlo como el tiempo más difícil frente a un 18,9 que ha contestado 'mal'. Los alumnos tenían que poner un ejemplo de verbo conjugado en este tiempo: un 38,1\% lo han hecho bien frente a un $11,9 \%$ que lo han hecho 'mal'.

La siguiente pregunta ha sido sobre el tiempo más fácil morfológicamente para un extranjero. El porcentaje de la respuesta correcta es muy bajo: 1,3\% (Imperfecto de Indicativo). Creemos que esto revela un profundo desconocimiento del sistema verbal español, además de una falta de reflexión y de conciencia metalingüística en nuestros estudiantes, que al no haber tenido formación lingüística específica sobre la enseñanza del español a alumnos no nativos, no son capaces de observar su propia lengua desde fuera. 
A continuación presentamos todos los resultados de esta pregunta (Tabla 5).

Tabla 5. Respuestas a la pregunta sobre el tiempo verbal más fácil morfológicamente.

\begin{tabular}{|l|c|}
\hline \multicolumn{1}{|c|}{ Tiempo verbal } & Porcentaje \% \\
\hline Formas simples de Indicativo & 25,2 \\
\hline 1 = Presente de Indicativo ('hablo') & 3,1 \\
\hline 2 = Futuro simple de Indicativo ('hablaré') & 0,9 \\
\hline 3 = Pretérito perfecto simple (Indefinido) de Indicativo ('hablé') & 1,3 \\
\hline 4 = Pretérito Imperfecto de Indicativo ('hablaba') & - \\
\hline $5=$ Condicional simple de Indicativo ('hablaría') & 0,6 \\
\hline Formas compuestas de Indicativo & - \\
\hline $6=$ Pretérito Perfecto Compuesto de Indicativo ('he hablado') & 0,6 \\
\hline $7=$ Pretérito Pluscuamperfecto de Indicativo ('había hablado') & - \\
\hline $8=$ Pretérito anterior de Indicativo ('hube hablado') & - \\
\hline $9=$ Futuro perfecto o compuesto de Indicativo ('habré hablado') & 0,6 \\
\hline $10=$ Condicional compuesto o perfecto Indicativo ('habría hablado') & - \\
\hline Formas simples de Subjuntivo & - \\
\hline $11=$ Presente de Subjuntivo ('hable') & \\
\hline $12=$ Pretérito imperfecto de Subjuntivo ('hablara' o 'hablase') & 0,3 \\
\hline $13=$ Futuro de Subjuntivo ('hablare') & - \\
\hline Formas compuestas de Subjuntivo & - \\
\hline $14=$ Pretérito perfecto compuesto de Subjuntivo ('haya hablado') & - \\
\hline $15=$ Pretérito pluscuamperfecto de Subjuntivo ('hubiera/se hablado') & 7,9 \\
\hline $16=$ Futuro Perfecto de Subjuntivo ('hubiere hablado') & \\
\hline $\begin{array}{l}17=\text { Modo Imperativo } \\
\text { 'habla', 'hablad' }\end{array}$ & \\
\hline $18=$ otro (mal; nombre inexistente de tiempo verbal) & \\
\hline
\end{tabular}

Un 38,4\% ha contestado 'bien' la pregunta sobre las razones para considerarlo como el tiempo más fácil frente a un 12,9\% que ha contestado 'mal' (pero recordemos que se equivocan de tiempo). Los alumnos tenían que poner un ejemplo de verbo conjugado en este tiempo: un 34,6\% lo ha hecho bien frente a un $6 \%$ que lo ha hecho 'mal'; casi el $60 \%$ no ha contestado.

La pregunta sobre los tiempos verbales es muy importante en el estudio y por eso, además del análisis descriptivo de frecuencia proporcionado anteriormente, hemos elaborado también tablas personalizadas en SPSS para comparar los resultados en función de la titulación de los estudiantes. Las hemos aplicado solo a las respuestas correctas para ver qué porcentaje tienen en el Grado de Educación Primaria y de Filología española. En cuanto al tiempo más fácil, hemos añadido también el Presente de Indicativo por ser la moda en el análisis de frecuencia, a pesar de no ser la respuesta correcta (Tabla 6). 
Tabla 6. Porcentaje de las respuestas correctas o de la moda sobre los tiempos verbales, según la titulación. Resultados con porcentaje de la fila.

\begin{tabular}{|l|l|c|c|}
\hline \multicolumn{2}{|c|}{ Pregunta } & Educación Primaria & Filología española \\
\hline \multirow{2}{*}{ Tiempo más difícil } & Presente de Indicativo & $35,6 \%$ & $6,5 \%$ \\
\cline { 2 - 4 } & $\begin{array}{l}\text { Pretérito Perfecto Simple } \\
\text { de Indicativo }\end{array}$ & $6,2 \%$ & $12 \%$ \\
\hline \multirow{2}{*}{ Tiempo más fácil } & Imperfecto de Indicativo & $0 \%$ & $3,3 \%$ \\
\cline { 2 - 4 } & Presente de Indicativo & $32,9 \%$ & $6,5 \%$ \\
\hline
\end{tabular}

Consideramos que una preparación básica para enseñar el español a extranjeros incluye el conocimiento sobre las funciones comunicativas y por eso hemos planteado tres preguntas al respecto en las que se pedían también tres ejemplos: ¿qué funciones comunicativas trabajarías con inmigrantes en A1, B1 y con inmigrantes adultos de nivel inicial? Ha sido una pregunta con trampa para los estudiantes, puesto que las funciones comunicativas en A1 y en B1 serán las mismas, por ejemplo, saludar, disculparse, agradecer, presentar a alguien, despedirse, dar y pedir información, expresar opiniones, y actitudes, expresar gustos, deseos y sentimientos, dar una orden o instrucción, pedir un favor, pedir ayuda, proponer y sugerir, ofrecer e invitar, aceptar o rechazar una invitación, etc.

En general, a medida que se avanza en los niveles va aumentando el número de funciones, así como el número de exponentes y su complejidad (se pueden comparar las funciones de 'rogar', 'amenazar' o 'responder a un saludo'). Por ejemplo, la función 'formular buenos deseos' se presenta en A2 con un exponente fijo 'que aproveche' que el alumno aprende de memoria, mientras que en B1, donde se ha estudiado el presente de subjuntivo, para la misma función se introduce 'que + presente de subjuntivo'.

Desde el nivel A1 hasta el B1, a pesar de que hay progreso, los recursos lingüísticos son limitados y esto se traduce en una capacidad limitada de creación lingüística. A partir del nivel B2 el aprendiente dispone de un abanico más amplio de recursos que le permite ser creativo con las formulaciones más abiertas de los exponentes. Ilustremos esto con un ejemplo del Instituto Cervantes (2007: 209) sobre la función de 'expresar deseos':

En A1: 'Quiero' + SN /inf.

En B1: 'Quiero que' + pres. subj.

En B2: 'Quería' / 'Querría' / ‘Quisiera' + subj.

[formulación más abierta que en B1, equivalente a 'Quiero' / 'Querría', etc. + todos los tiempos del subjuntivo]

En C1: 'Me muero de ganas de / por'... 
[formulación más abierta que en B2, equivalente a = 'me muero de ganas de / por' + todas las combinaciones posibles: 'verte', 'que vengas', etc. ]

Otra observación que hemos de destacar es que la mayor parte de los exponentes responden a muestras de lengua oral y a un registro neutro o estándar hasta B1. La inclusión de las marcas de registro (coloquial, formal, pero sin el vulgar) se inicia en B2 y aumenta en C1 y C2 (Instituto Cervantes, 2007).

En cuanto a la pregunta sobre las funciones para inmigrantes adultos del nivel inicial, serían las mismas que ya hemos comentado, pero nos gustaría comentar la propuesta de Villalba Martínez y Hernández García (2002) quienes prevén doce entornos comunicativos en los que explicitan una serie de funciones comunicativas con sus correspondientes exponentes gramaticales, léxicos y culturales: 1. Los papeles: documentos y trámites administrativos; 2 . La ciudad y el barrio: orientarse en la ciudad; 3. Los transportes públicos; 4. La salud; 5. Ir al mercado (la compra); 6. Compra de ropa y calzado; 7. Buscar piso (alquilar, contrato, etc.); 8. El trabajo; 9. Gustos y aficiones; 10. Vida cotidiana; 11. Planes para fines de semana o vacaciones y 12. El pasado y la infancia.

Según los resultados de nuestro estudio, los estudiantes del Grado de Primaria han contestado la pregunta sobre las funciones en A1 de la siguiente manera (Tabla 7): bien $(66,7 \%)$, regular $(17,3 \%)$ y mal $(4,4 \%)$. Los estudiantes de Filología española han obtenido los siguientes porcentajes: bien $(23,9 \%)$, regular $(31,5 \%)$ y mal $(3,3 \%)$.

Tabla 7. Funciones comunicativas.

\begin{tabular}{|l|c|c|c|c|c|c|c|c|c|}
\hline & \multicolumn{3}{|c|}{ ¿Qué funciones comunicativas trabajarías con inmigrantes en A1? Pon 3 } \\
ejemplos.
\end{tabular}

Se les pidió a los encuestados que pusieran tres ejemplos de funciones. Hemos agrupado las respuestas en la Tabla 8:

Tabla 8. Ejemplos de funciones.

\begin{tabular}{|l|c|c|c|}
\hline & bien & regular & mal \\
\hline Nivel A1 & $44,5 \%$ & $46,6 \%$ & $8,9 \%$ \\
\hline Nivel B1 & $44,5 \%$ & $44,5 \%$ & $10,9 \%$ \\
\hline Inmigrantes adultos del nivel inicial & $49,5 \%$ & $36,9 \%$ & $13,5 \%$ \\
\hline
\end{tabular}

Hemos planteado también la pregunta de en qué se va a diferenciar el discurso del profesor en el aula cuando tiene alumnos inmigrantes con insuficiente competencia 
lingüística en español. En esta pregunta esperamos que los estudiantes sean capaces de formular estos cambios en el discurso del profesor: hablar más lento, articular mejor, usar sinónimos con mayor frecuencia, parafrasear, simplificar el léxico y la sintaxis, evitar las oraciones largas, gesticular más, usar imágenes cuando es posible, formular preguntas para verificar la comprensión (tanto para inmigrantes como para nativos), etc.

Las respuestas de esta pregunta sobre los cambios en el discurso del profesor fueron agrupadas como: bien $(37,4 \%)$, regular $(10,4 \%)$ y mal $(1,3 \%)$.

Para los objetivos de la investigación nos planteamos también conocer la importancia que conceden los estudiantes a diferentes contenidos en la enseñanza del español como segunda lengua y han tenido que ordenarlos, asignando un número de 1 a 5 por orden de importancia de menor a mayor grado $(1=$ nada importante; $2=$ indiferente; $3=$ poco importante; $4=$ importante y $5=$ muy importante). En la Tabla 9 recogemos los porcentajes de las respuestas.

Tabla 9. Importancia de distintos contenidos en EL2.

\begin{tabular}{|l|l|c|c|c|c|c|}
\hline $\mathbf{N .}^{\mathbf{0}}$ & Contenidos & $\mathbf{1}$ & $\mathbf{2}$ & $\mathbf{3}$ & $\mathbf{4}$ & $\mathbf{5}$ \\
\hline 49.1 & Métodos de enseñanza de segundas lenguas & $3,3 \%$ & $6,2 \%$ & $22,9 \%$ & $28,4 \%$ & $39,2 \%$ \\
\hline 49.2 & Gramática del español & $0,7 \%$ & $4,6 \%$ & $14,4 \%$ & $27,1 \%$ & $53,3 \%$ \\
\hline 49.3 & Programación y diseño de unidades didácticas & $6,9 \%$ & $9,9 \%$ & $27,4 \%$ & $35,3 \%$ & $20,5 \%$ \\
\hline 49.4 & Comunicación no verbal & $2 \%$ & $12,9 \%$ & $26,7 \%$ & $32 \%$ & $26,4 \%$ \\
\hline 49.5 & Interculturalidad & $1,7 \%$ & $6,3 \%$ & $24,1 \%$ & $38 \%$ & $30 \%$ \\
\hline 49.6 & $\begin{array}{l}\text { TIC (Tecnologías de la Información y la } \\
\text { Comunicación) }\end{array}$ & $4,3 \%$ & $12,7 \%$ & $27,3 \%$ & $37 \%$ & $18,7 \%$ \\
\hline 49.7 & $\begin{array}{l}\text { Datos demográficos sobre la población } \\
\text { inmigrante }\end{array}$ & $14,2 \%$ & $26,8 \%$ & $34,8 \%$ & $17,9 \%$ & $6,3 \%$ \\
\hline 49.8 & $\begin{array}{l}\text { Datos etnográficos, culturales y sociales sobre } \\
\text { países de procedencia }\end{array}$ & $6,8 \%$ & $11,4 \%$ & $34,9 \%$ & $34,5 \%$ & $12,4 \%$ \\
\hline 49.9 & $\begin{array}{l}\text { Legislación española sobre inmigración y } \\
\text { extranjería }\end{array}$ & $14,2 \%$ & $19,9 \%$ & $27,2 \%$ & $26,8 \%$ & $11,9 \%$ \\
\hline
\end{tabular}

Se observa que se le concede mayor importancia a la gramática (53,3\% 'muy importante'), seguida por 'métodos de enseñanza de segundas lenguas' (39,2\%).

En el cuestionario se formuló también una pregunta sobre la principal dificultad que le surgiría a un inmigrante para aprender el español. Los sujetos tenían que elegir solo una opción. Los resultados (Gráfico 2) indican por orden descendente: gramática $(50,3 \%)$, producción oral $(23,2 \%)$, pronunciación $(15,4 \%)$, escritura $(8,1 \%)$ y lectura $(3 \%)$. 
50. ¿Cuál crees que será la principal dificultad que le surgirá a un inmigrande para aprender el español? Elige solo una opción



Gráfico 2. Dificultad principal para un inmigrante.

Nos interesa también conocer qué importancia se le concede a distintos factores que causan las dificultades para aprender una segunda lengua. Los sujetos han tenido que ordenarlos, asignando un número de 1 a 5 por orden de importancia de menor a mayor grado. En la Tabla 10 se presentan los porcentajes de las respuestas.

Tabla 1o. Importancia de distintos factores que causan dificultades en el aprendizaje de EL2.

\begin{tabular}{|c|l|c|c|c|c|l|}
\hline $\mathbf{N .}^{\mathbf{0}}$ & \multicolumn{1}{|c|}{ Factores } & $\mathbf{1}$ & $\mathbf{2}$ & \multicolumn{1}{|c|}{$\mathbf{3}$} & \multicolumn{1}{|c|}{$\mathbf{4}$} & \multicolumn{1}{|c|}{$\mathbf{5}$} \\
\hline 52.1 & La edad & $7,3 \%$ & $20,3 \%$ & $11,3 \%$ & $32,6 \%$ & $28,6 \%$ \\
\hline 52.2 & Nivel socioeconómico & $14 \%$ & $31,3 \%$ & $19 \%$ & $21,7 \%$ & $14 \%$ \\
\hline 52.3 & Formación previa y nivel cultural & $1,7 \%$ & $21,3 \%$ & $5 \%$ & $37,2 \%$ & $34,9 \%$ \\
\hline 52.4 & $\begin{array}{l}\text { Interés y motivación relacionada con las } \\
\text { necesidades }\end{array}$ & $0,7 \%$ & $10,3 \%$ & $1,7 \%$ & $28,9 \%$ & $58,5 \%$ \\
\hline 52.5 & $\begin{array}{l}\text { Oportunidades para usar la lengua y frecuencia } \\
\text { de uso }\end{array}$ & $1,3 \%$ & $8,3 \%$ & $3,7 \%$ & $39,2 \%$ & $47,5 \%$ \\
\hline 52.6 & Diferencias lingüísticas con su lengua materna & $3,4 \%$ & $22,8 \%$ & $6,7 \%$ & $35,2 \%$ & $31,9 \%$ \\
\hline
\end{tabular}

\subsection{Resultados del análisis inferencial}

Se aplicó esta técnica de análisis para establecer la relación de dependencia significativa entre variables. Se incluyen algunos de los resultados.

Se ha confirmado una relación significativa entre la titulación que está cursando el estudiante y otras variables que detallamos a continuación. 
Existe asociación significativa entre la titulación (P2) y señalar el tiempo verbal más fácil morfológicamente para un inmigrante: $x^{2}(18)=124,007, p<, 05$; test exacto de Fisher, $\mathrm{F}=, 002$. La fuerza de asociación, medida con el coeficiente $\mathrm{V}$ de Cramer, indicó una dependencia media $(\mathrm{V}=, 442)$.

Se confirmó también la relación significativa entre la titulación y el conocimiento de en qué consiste una adaptación curricular: $x^{2}(4)=162,945, p<, 05$. La fuerza de asociación, medida con el coeficiente $\mathrm{V}$ de Cramer, indicó una dependencia media $(\mathrm{V}=, 506)$.

En la Tabla 11 presentamos algunos de los resultados del análisis inferencial con tablas de contingencia.

Tabla 11. Resultados del análisis inferencial.

\begin{tabular}{|c|c|c|c|c|c|c|}
\hline Variables & $\begin{array}{c}\text { Chi- } \\
\text { cuadrado } \\
x^{2}\end{array}$ & $\begin{array}{c}\text { Grado } \\
\text { de } \\
\text { libertad }\end{array}$ & $\begin{array}{l}\text { p valor del } \\
\text { chi- } \\
\text { cuadrado }\end{array}$ & $\begin{array}{c}\text { Test } \\
\text { exacto } \\
\text { de } \\
\text { Fisher }\end{array}$ & $\begin{array}{c}\text { V de } \\
\text { Cramer }\end{array}$ & $\begin{array}{c}\text { Grado de } \\
\text { dependencia }\end{array}$ \\
\hline $\begin{array}{l}\text { Dependencia entre } \\
\text { la titulación y señalar } \\
\text { el tiempo verbal más } \\
\text { fácil }\end{array}$ & 124,007 & 18 & ,002 & ,002 &, 568 & medio \\
\hline $\begin{array}{l}\text { Dependencia entre } \\
\text { la titulación y el } \\
\text { conocimiento de } \\
\text { adaptaciones } \\
\text { curriculares }\end{array}$ & 162,945 & 4 & ,000 & ,000 & , 481 & medio \\
\hline $\begin{array}{l}\text { Dependencia entre } \\
\text { la titulación (P2) y la } \\
\text { importancia de la } \\
\text { programación y } \\
\text { diseño de unidades } \\
\text { didácticas }\end{array}$ & 262,279 & 10 &, 000 & & ,642 & medio \\
\hline $\begin{array}{l}\text { Entre la titulación y } \\
\text { las funciones } \\
\text { comunicativas en A1 }\end{array}$ & 202,884 & 6 & ,000 & &, 565 & medio \\
\hline $\begin{array}{l}\text { Entre la titulación y } \\
\text { las aulas ATAL }\end{array}$ & 130,131 & 6 & ,000 & & , 452 & medio \\
\hline
\end{tabular}

En cuanto a las preguntas sobre los tiempos verbales, el $84,6 \%$ de los estudiantes de Filología española seleccionaron correctamente el tiempo verbal más difícil, porcentaje que fue significativamente mayor al $59,5 \%$ de respuestas correctas de los estudiantes de Grado de Educación Primaria $\left(\chi^{2}(1)=13,920, V=0,268, p<0,001\right)$.

Sin embargo, no detectamos diferencias significativas a la hora de razonar sobre la selección del tiempo verbal más fácil, con un $75,4 \%$ de respuestas correctas para los estudiantes de Grado de Educación Primaria y un 85,7\% de respuestas correctas en estudiantes de Filología española $\left(x^{2}(1)=3,026, V=0,125, p=0,103\right)$. 
No se detectaron diferencias significativas en el número de respuestas correctas para el tiempo verbal más fácil $\left(x^{2}(3)=2,511, \mathrm{~V}=0,113, \mathrm{p}=0,476\right)$ ni para el tiempo verbal más difícil $\left(x^{2}(3)=2,710, \mathrm{~V}=0,118, \mathrm{p}=0,442\right)$ al comparar los sujetos por curso.

Detectamos una relación significativa entre la titulación que está cursando el sujeto entrevistado y la definición de las aulas $\operatorname{ATAL}\left(x^{2}(2)=14,790, \mathrm{~V}=0,252, \mathrm{p}=0,001\right)$, donde se observa que los sujetos del Grado de Educación Primaria responden principalmente 'aulas de adaptación lingüística' (45,9\%) mientras que los sujetos de Filología española respondieron principalmente 'aulas temporales de asesoramiento legal para alumnos inmigrantes' $(68,9 \%)$.

Otro resultado del análisis inferencial indica que el desconocimiento de las aulas ATAL es significativamente mayor entre los estudiantes de Filología española $(92,9 \%)$ que en los de Grado en Educación Primaria $\left(75,2 \% ; x^{2}(1)=12,081, V=0,20, p=0,001\right)$.

\section{CONCLUSIONES}

El estudio confirma el desajuste existente entre la formación de los futuros docentes y la demanda laboral o la realidad laboral, en la que se van a enfrentar a las dificultades y diferencias específicas en la atención al alumnado extranjero en comparación con el alumnado nativo. La diversidad en las aulas es un hecho y quizás los estudiantes universitarios tengan alguna formación en atención a la diversidad, en interculturalidad y en educación inclusiva, en general, pero siguen padeciendo una carencia notable en la formación específica para enseñar español a alumnos inmigrantes que difiere mucho de lo que supone la enseñanza de Lengua a nativos. Creemos que se trata de una laguna que urge subsanar, porque la competencia en español como lengua vehicular mejorará el rendimiento académico de los alumnos inmigrantes y, además, la competencia comunicativa en español es la vía más eficaz para la integración social y cultural de este colectivo.

Como hemos constatado, existe una deficiencia formativa en este ámbito en asignaturas clave como la Lingüística Aplicada, en temas como la adquisición de la Lengua Materna (L1) y la Lengua Extranjera (L2), o la Didáctica de Lenguas Extranjeras, en aspectos como los enfoques y métodos en la enseñanza de lenguas extranjeras, así como en diseño curricular.

Finalmente, para solucionar esta situación, entendemos que deben realizarse modificaciones curriculares en las titulaciones analizadas, mediante la inclusión de los temas indicados en dichas asignaturas, todo ello en el marco de una política global de atención a la población inmigrante. Dichas modificaciones, como señalan algunos autores de referencia, deberían ser impulsadas por las instituciones públicas generales del Estado y de las CC.AA. 
Como limitaciones y líneas de mejora, debemos señalar que este estudio ha sido acompañado de otro cuestionario, parecido pero no igual, que fue contestado por profesores de español en todos los ámbitos educativos: Universidad, Educación Primaria y Secundaria, Escuelas Oficiales de Idiomas, profesores en ATAL, en academias y varias ONG. El segundo cuestionario se analiza aparte, por cuestiones de espacio en un artículo de revista, pero quizás sea interesante contrastar los resultados de los dos, algo que nos planteamos hacer en el futuro.

El instrumento de investigación ha permitido usar dos técnicas estadísticas: análisis descriptivo de frecuencia y análisis inferencial. En una investigación futura diseñaríamos un instrumento que permitiera sacar más jugo a los datos, a pesar de que el nuestro nos ha permitido conseguir los objetivos de la investigación. Creemos que una investigación futura que sirva de ampliación se puede realizar en un grupo de control y otro, experimental, para poder contrastar los resultados con y sin instrucción. Nos parece interesante, porque confirmaríamos de una manera todavía más convincente la necesidad de formación específica para enseñar el español a inmigrantes.

\section{REFERENCIAS BIBLIOGRÁFICAS}

ANECA (2005). Libro Blanco. Titulo de Grado en estudios en el Ámbito de la Lengua, Literatura, Cultura y Civilización [en línea]. Disponible en: https://bit.ly/2QZJIPE

Cruz Piñol, M. (2004). La formación de profesores de ELE desde las licenciaturas de Filología. En E. Martinell (Coord.), La oferta formativa del profesorado de E/LE (pp. 19-30). Madrid: Edinumen.

García, J. A., Sánchez, P., Moreno, I. \& Goenechea, C. (2010). Estudio del sistema y funcionamiento de las aulas de enlace de la Comunidad de Madrid. De la normativa institucional a la realidad cotidiana. Revista de Educación, 352, 473493.

Grañeras, M., Vázquez, E., Parra, A., Rodríguez, F., Madrigal, A. \& Vale, P. (2007). La atención lingüística al alumnado extranjero en el sistema educativo español: Normativa, actuaciones y medidas. Revista de Educación, 343, 149-174.

Gutiérrez, R. \& Miyar, M. (2010). Conocimiento del español e integración social de los inmigrantes. En J. A. Alonso \& R. Gutiérrez (Dirs.), Emigración y lengua. El papel del español en las migraciones internacionales (pp. 161-227). Madrid: Ariel.

Instituto Cervantes (2007). Plan curricular del Instituto Cervantes. Madrid: Biblioteca Nueva. 
Mato, J. \& Gutiérrez, R. (2010). Logros laborales de los inmigrantes económicos en España: El papel de la lengua española. En J. A. Alonso \& R. Gutiérrez (Dirs.), Emigración y lengua. El papel del español en las migraciones internacionales (pp. 101-159). Madrid: Ariel.

Moreno García, C. (2007). La enseñanza y el aprendizaje del español (castellano) en aulas multilingües. De los fundamentos a las expectativas. Revista de Educación, $343,15-33$.

Níkleva, D. \& Ortega Martín, J. L. (2015). La formación del alumnado del Grado de Educación Primaria para enseñar a alumnos inmigrantes y las medidas de intervención educativa. Cultura y Educación, 27(2), 316-330.

OECD (2016). Trends Shaping Education 2016. OECD Publishing, Paris [en línea]. Disponible en: https://dx.doi.org/10.1787/trends_edu-2016-en

Pastor Cesteros, S. (2007). Enseñar una segunda lengua (SL) y en una SL: Hacia una nueva formación del profesorado de español para inmigrantes. Linred. Lingüistica en la red, monográfico, 1-10.

Rico-Martín, A. M. \& Níkleva, D. (2017). La enseñanza del español como segunda lengua en la formación universitaria de los futuros docentes: Análisis de planes de estudios y percepción de los estudiantes. En D. Níkleva (Ed.), La formación de los docentes de español para inmigrantes en distintos contextos educativos (pp. 163-195). Bern: Peter Lang.

Villalba Martínez, F. \& Hernández García, Ma T. (2002). Programación para la enseñanza del español como L2 a adultos inmigrantes [en línea]. Disponible en: https://bit.ly/2PFAFcJ

Villalba Martínez, F. \& Hernández García, Ma T. (2008). La enseñanza del español a inmigrantes en contextos escolares. En J. Sánchez Lobato \& I. Santos Gargallo (Eds.), Vademécum para la formación de profesores. Enseñar el español como segunda lengua (L2)/lengua extranjera (LE) (pp. 1225-1258). Madrid: SGEL.

Villalba Martínez, F. \& Hernández García, Ma T. (2010). Perspectivas y líneas de trabajo en la enseñanza de español a inmigrantes. Monográfico, MarcoELE, 10, 163-184.

\section{NOTAS}

${ }^{1}$ La investigación se realiza en el marco de un proyecto I+D (referencia: EDU2013-43868-P), titulado "La formación de los docentes de español para inmigrantes en diferentes contextos educativos". 
2 Según los datos del Instituto Nacional de Estadística para 2017 (accesibles en https://bit.ly/2PZUm2U), aunque se observa una disminución en el número de inmigrantes desde 2008, pero a partir de 2013 las cifras vuelven a crecer, produciéndose un notable aumento en 2017 (174231 personas).

3 Se evidencia que los inmigrantes presentan bajos rendimientos, constatándose que las diferencias de rendimiento entre los estudiantes inmigrantes y los nativos puede llegar a ser de 52 puntos a favor de los nativos. Del mismo modo, el colectivo de estudiantes inmigrantes presenta un mayor índice de abandono, el 43,6 \% (OECD, 2016). Ante esta situación, diversos estudios confirman la importancia del desarrollo de la competencia lingüística de los inmigrantes como factor determinante para facilitar la integración social, educativa y el acceso al mercado laboral de estas personas (Gutiérrez \& Miyar, 2010; Mato \& Gutiérrez, 2010).

En este sentido, debemos ser conscientes de que, tal y como afirman Villalba y Hernández (2010: 166), "la situación de inmigración no garantiza por sí misma la exposición continuada a la L2. Es más, habrá casos en los que el no nativo apenas utilizará la nueva lengua, desarrollando el conjunto de sus interacciones cotidianas en su LM”.

${ }^{4}$ El cuestionario está disponible en el siguiente enlace: https://bit.ly/2S60aWj 